\title{
Utilizing Genomic Selection for Wheat Population Development and Improvement
}

\author{
Lance F. Merrick ${ }^{1}$, Andrew W. Herr ${ }^{1}$, Karansher S. Sandhu ${ }^{1}$, Dennis N. Lozada ${ }^{2}$, Arron H. Carter ${ }^{1 *}$
}

1 Department of Crop and Soil Sciences, Washington State University, Pullman, WA 99164, USA; lance.merrick@wsu.edu (L.F.M.): andrew.herr@wsu.edu (A.W.H.); k.sandhu@wsu.edu (K.S.S);

2 Department of Plant and Environmental Sciences, New Mexico State University, Las Cruces, NM 88003, USA; dlozada@nmsu.edu (D.N.L)

* Correspondence: ahcarter@wsu.edu; Tel.: +1-509-335-6198

\begin{abstract}
Wheat (Triticum aestivum L.) breeding programs can take over a decade to release a new variety. However, new methods of selection such as genomic selection (GS) must be integrated to decrease the time it takes to release new varieties to meet the demand of a growing population. The implementation of GS into breeding programs is still being explored, with many studies showing its potential to change wheat breeding through achieving higher genetic gain. In this review, we explore the integration of GS for a wheat breeding program by redesigning the traditional breeding pipeline to implement GS. We propose implementing a two-part breeding strategy by differentiating between population improvement and product development. The implementation of GS in the product development pipeline can be integrated into most stages and can predict within and across breeding cycles. Additionally, we explore optimizing the population improvement strategy through GS recurrent selection schemes to reduce crossing cycle time and significantly increase genetic gain. The recurrent selection schemes can be optimized for parental selection, maintenance of genetic variation, and optimal cross-prediction. Overall, we outline the ability to increase the genetic gain of a breeding program by implementing GS and a two-part breeding strategy.
\end{abstract}

Keywords: Plant Breeding; Two-Part Strategy; Recurrent Selection; Population Improvement; Product Development; Optimization; Genetic Gain; Cross-Prediction

\section{Wheat breeding}

Wheat (Triticum aestivum L.) is one of the essential cereal grain crops and is the principal cereal grain used for food consumption in the US and most of the world [1]. Yet, the world population is projected to reach 9 billion people by 2050, and to account for this growing demand for food, production systems will have to increase productivity by $50 \%$ while facing a decrease in agricultural land [2]. Increasing productivity of food systems partially relies on developing improved cultivars. However, it usually takes 11 to 12 years to develop new wheat cultivars [3]. Traditionally, after crossing and population development, inbred lines are developed either through self-pollination or doubled haploids. The inbred lines are then phenotyped in headrows and field trials before being selected as parents in the crossing block. This method takes up to four to six years in wheat, depending on the breeding program structure and preference of the breeder. In the Washington State University Winter Wheat breeding program, for example, the inbred lines are developed through both self-pollination and doubled-haploid production. Headrows are the first stage of phenotyping and happens in the fourth year, followed by unreplicated preliminary yield trials (PYT) in the fifth year. In the sixth year, replicated field trials begin at multiple locations, and move to state-wide advanced yield trials (AYT) at additional locations to screen for a variety of traits. Inbred lines can be in replicated yield trials up to five or more years by the time varieties are ultimately released from the breeding program. 
Therefore, modern breeding approaches, which can reduce cycle time and increase selection precision, along with the efficient use of genetic variation, can be exceedingly important to increase genetic gains [2].

\section{Genomic selection}

One modern breeding approach, genomic selection (GS), is posed to increase genetic gains and reduce cycle time for complex agronomic traits, such as grain yield and disease resistance [4,5]. Meuwissen et al. [6] proposed the idea of simultaneously estimating all markers regardless of "significance" and thereby capturing all marker's effects, and coined this method "Genomic Selection." The goal of GS is to calculate genomic estimated breeding values (GEBVs). GS is accomplished by using a population of individuals with both phenotypic and genotypic data called the training population. The training population is used to create a prediction model to simultaneously estimate allele effects at all loci. A statistical model is created or trained on the training set to estimate model parameters that are used to calculate GEBVs of individuals with only genotypic data, called the test population [7]. The selection for the advancement in the breeding program is then based on the GEBVs of the lines rather than phenotypic selection (PS), hence the term "Genomic Selection".

As an approach, GS can improve a breeding program by increasing genetic gain and enhancing trait selection $[5,8,9]$. Genetic gain, also known as the genetic response $(R)$, is calculated by what is known as the breeder's equation, $R=\frac{i r \sigma_{A}}{t}$, where $i$ is the selection intensity; $\sigma_{A}$ is the square root of the additive genetic variance; $r$ is the selection accuracy, which is the equivalent to narrow-sense heritability $\left(h^{2}\right)$ in PS; and $t$ is the cycle time $[10,11]$. Plant breeders use this equation to increase the genetic gain of their breeding program through subsequent cycles of selection. By increasing one of the components in the numerator (selection intensity or selection accuracy) or decreasing cycle time, a breeder can increase genetic gain [11]. GS reduces the length of the breeding cycle by selecting based on GEBVs as opposed to phenotypes. Previously, Lorenzana and Bernardo [12] showed a response per cycle for GS was half that of PS. However, GS can also be implemented in earlier generations, which can be a substitute for phenotyping that is expensive or time-intensive. Thus, selections can be made in a shorter timeframe and reduced cycle time [8]. In contrast, Rutkoski et al. [5] displayed that PS and GS exhibited similar genetic gain. However, GS reduced genetic variance, which indicated that maintaining genetic variance needs to be accounted for in a GS breeding pipeline.

Both PS and marker-assisted selection (MAS) are effective for increasing genetic gain for high heritability traits [7]. Highly heritable traits such as disease resistance can be selected for early in the breeding program with high accuracy using MAS. Even though MAS has helped improve plant breeding and increased genetic gain, it is less effective when used for polygenic and complex traits. Additionally, the increase of genetic gain using PS is also difficult for complex traits with low heritability [5]. Consequently, selection for important traits with low heritability, such as grain yield, is completed at the later stages of a breeding program. If the environmental effect, such as drought, disease pressure, or other adverse conditions, is high enough, accurate PS will be challenging. In contrast, GS can still be used when PS and MAS are ineffective, such as selecting for grain yield in early generation lines. It can increase gain per unit time, increase selection for difficult to measure traits, or assess the performance of individuals in environments where they have not been phenotyped. Further, GS can reduce the amount of phenotyping that is completed when there are too many lines to phenotype, and when seed amounts are insufficient [13].

The implementation of GS into breeding programs is still being explored. Many studies have shown the potential of GS to change traits through higher genetic gain $[4,5,14,15]$. Wheat breeding programs are still determining the best strategy to implement GS within the pipeline [16]. Reviews have helped guide the integration of GS within wheat breeding 
programs $[2,17,18]$. As genotyping costs continue to decrease, GS is starting to be practically cheaper than PS. Nevertheless, various aspects need to be considered first before integrating GS in the breeding pipeline. In order to implement GS and replace PS, breeding programs have to account for selecting on GEBVs instead of phenotypic values, the time it takes for genotyping and DNA extraction, and the use of doubled-haploids (DH) in rapid-cycle recurrent GS schemes. Also, previous GS studies focused on selecting lines based exclusively on GEBV; however, the implementation of phenotypic validation of the top selections still needs to be made since most prediction accuracies only account for a portion of the variance [16]. Genomic selection allows the use of GEBVs in lieu of phenotypic data which allows the restructuring of the traditional breeding program. Gaynor et al. [19] proposed reorganizing the traditional breeding program into two parts: the product development (PD) component, which is similar to traditional breeding programs, and a population improvement (PI) component to utilize recurrent GS. In this review, we explore the integration of GS for a wheat breeding program by redesigning the traditional breeding pipeline to differentiate between PD and PI while optimizing a two-part strategy based on the components of the breeder's equation.

\section{Product development}

The PD part of wheat breeding programs focuses on developing inbred lines for release as inbred varieties. The PD pipeline is the same as a traditional breeding program and therefore is the easiest and most flexible component to change. One of the major changes between the PD pipeline and the traditional breeding program is that lines from PD are not necessarily chosen for population improvement. However, the lines phenotyped in PD will need to be genotyped to update the training population for GS implementation. Stochastic simulations were previously used to compare long-term selection and genetic gain using various breeding program structures [19]. Gaynor et al. [19] simulated a two-part breeding program that used GS to conduct recurrent selection on F1 plants for PI and additional parental selection in PYTs and a similar program with parental selection in headrows (Figure 1). The two-part breeding programs showed up to 2.47 times the genetic gain than the conventional program with no GS, and up to 1.46 times the genetic gain relative to the conventional program with GS alone but no parental selection. Overall, the two-part strategy improved the genetic gain of a breeding program at similar costs and resource allocation. 


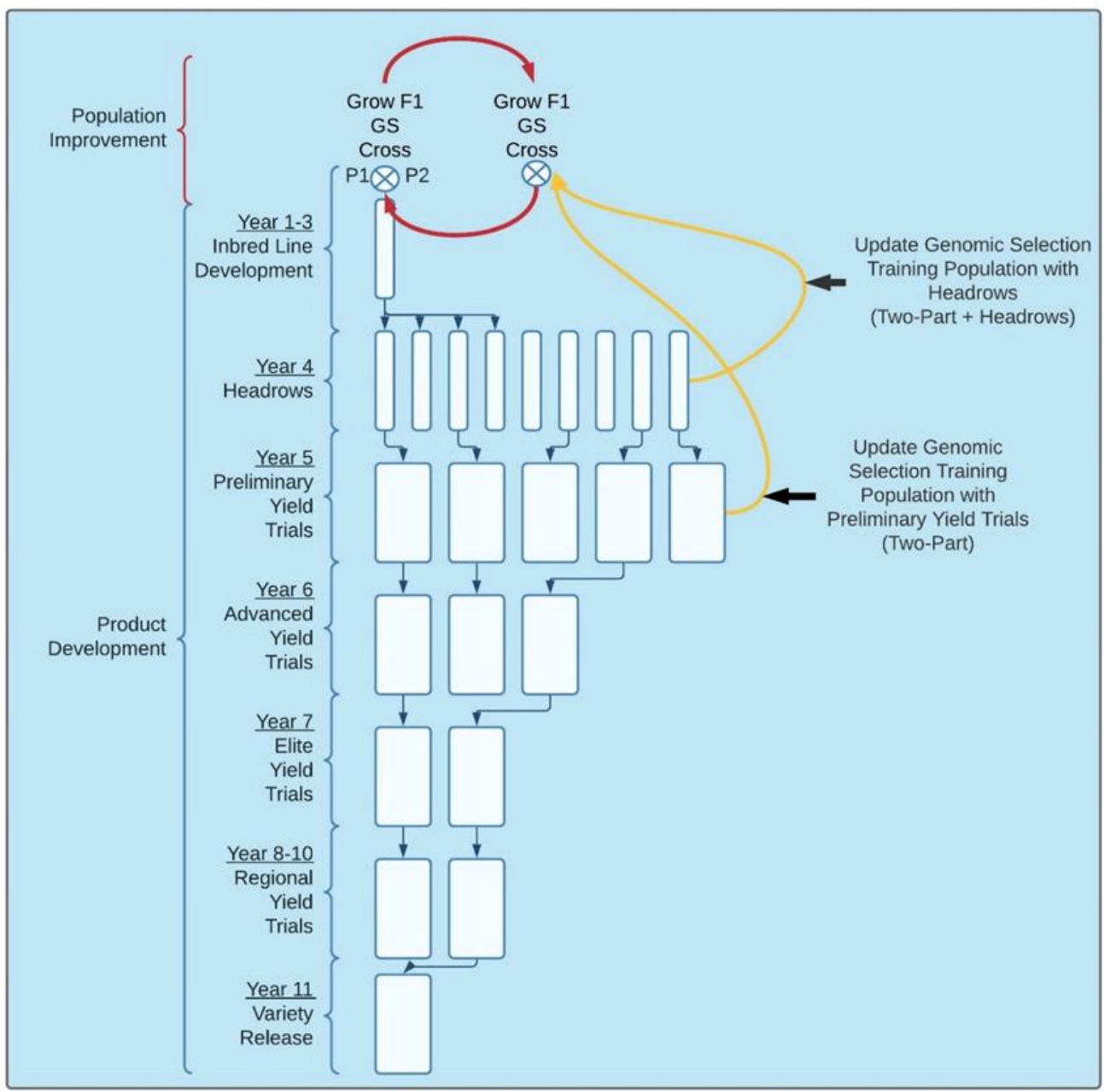

Figure 1. Overview of the two-part breeding strategy based on an 11-year breeding program from parental crossing to variety release. The recurrent selection scheme is shown in red arrows using $F_{1 s}$ for continuous crossing within-population improvement, whereas the blue lines show the product development (PD) pipeline. The yellow arrows display the possible implementations of GS into the PD pipeline for integration into the recurrent selection scheme, which include utilizing lines from the preliminary yield trials (TwoPart) or lines from headrows (Two-Part + Headrows) to update the GS training population.

\subsection{Implementation of GS for recurrent and parental selection}

Replacing phenotypes with GEBVs allows the restructuring of breeding programs. Genomic selection can simply replace phenotypic or MAS for selection purposes $[14,20]$. However, this strategy does not necessarily increase genetic gain for certain traits, such as grain yield, due to the lack of increase in selection accuracy compared to PS. Therefore, breeders need to determine the best stage to implement GS to increase every aspect of the breeder's equation and maximize genetic gain. GS can be implemented at any stage in order to select lines for the next stage in the breeding program. In general, field trials begin with many lines with fewer replications and environments, then the number of lines is sequentially decreased while increasing replications in individual trials and more locations. There are several opportunities to increase the genetic gain by optimizing breeding programs for GS. These include reorganizing field designs, increasing the number of lines evaluated, and leveraging the large amount of genomic and phenotypic data collected 
across different growing seasons and environments to increase heritability estimates, selection intensity, and selection accuracy [16,21,22]. Optimization for GS implementation enhances genetic gain and can decrease costs.

For parental or recurrent selection in early generations, GS implemented as early as the $F_{1}$ or $F_{2}$ generations is effective without including a generation of PS (Figure 2) [16]. Implementing GS as early as possible displayed increases in genetic gain by nearly six times compared to phenotypic selection with no GS implementation [16]. However, implementing GS early increases genotyping costs, whereas using GS in later generations with one stage of PS, reduces the number of lines genotyped (Figure 2). The implementation of GS in the $\mathrm{F}_{3}$ and $\mathrm{F}_{4}$ generations displayed a two-fold increase in genetic gain in $\mathrm{F}_{3}$ selection compared to PS in the same stage [16]. Implementation in the F3 displayed a compromise between early implementation with no PS, and PS with GS implemented in late stage of the program (Figure 2). Implementing GS in the late stages of the breeding program was shown to have little advantage in increasing genetic gain than PS alone [16]. Additionally, Longin et al. [23] showed through simulation, that when genomic selection accuracy is low, one stage of GS should be followed by one stage of PS. Additionally, GS would be recommended only when accuracy is very high. In the low accuracy scenarios, GS would be useful with truncation selection for removing the lowest performing lines.

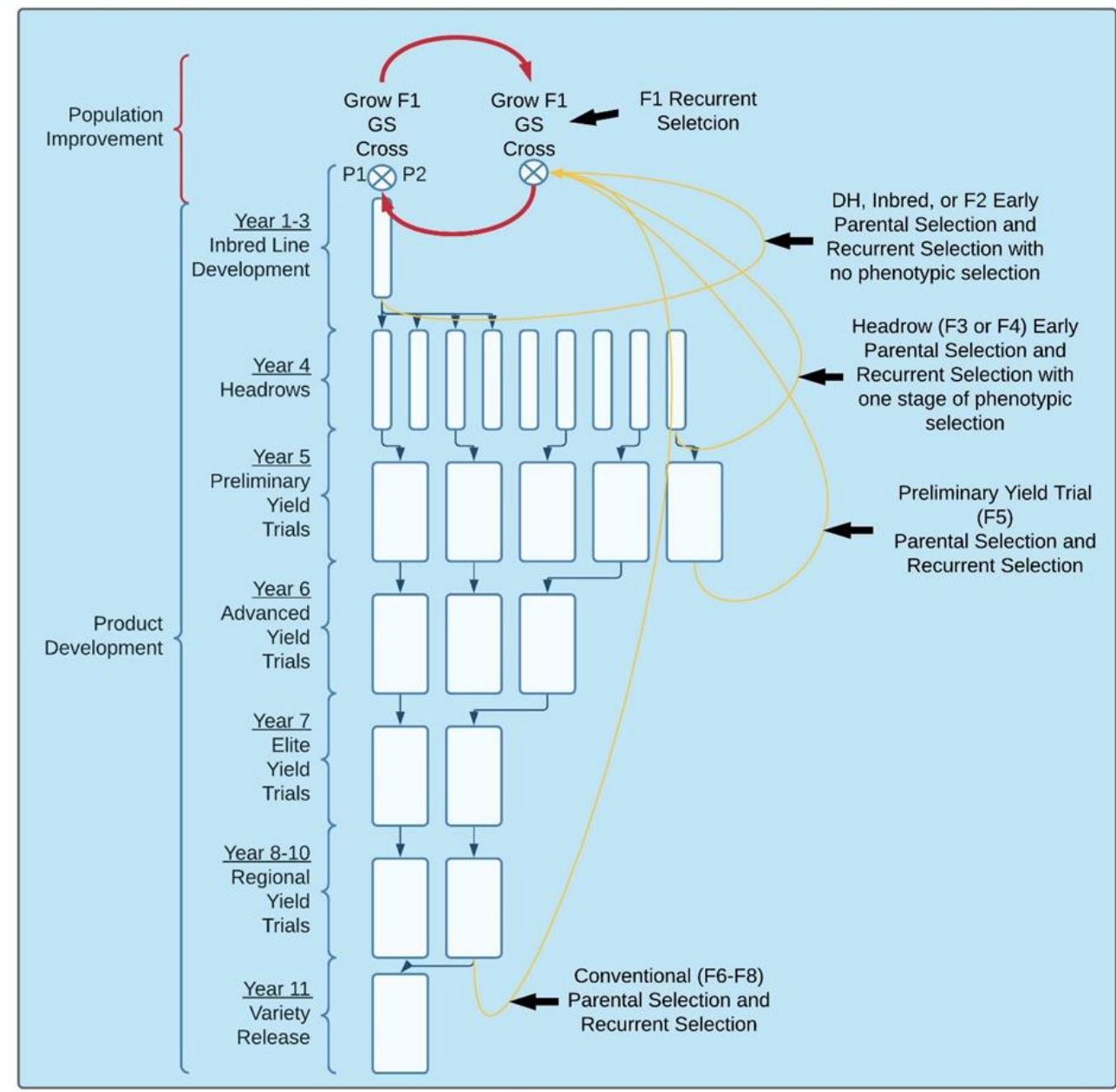

Figure 2. The implementation of GS for recurrent and parental selection based on an 11year breeding program from parental crossing to variety release within the two-part breeding strategy. The recurrent selection scheme is shown in red arrows using Fis for continuous crossing within-population improvement, whereas the blue arrows show the 
product development (PD) pipeline. The yellow arrows display the possible implementations of GS into the PD pipeline to select lines for integration into the recurrent selection scheme. GS can be implemented as early as the F2 stage, through doubled-haploid production or speed breeding without phenotypic screening. Further, GS can be implemented throughout the PD pipeline with an increasing number of phenotyping stages, from a single stage of phenotyping in headrows to the conventional method of phenotyping up to the 10th year in yield trials.

\subsection{Implementation of GS for within and across breeding cycles}

Genomic selection can also be implemented in the PD pipeline for across and within breeding cycles for selection and advancement (Figure 3) [9,15,24,25]. Early generation GS is superior to conventional PS in line breeding and can be strongly improved by including additional information from later generation PYTs and AYTs [9,25]. For instance, phenotypic data from the PYT or AYT can be used to predict advancements across breeding cycles as early as F2, DH, and inbred lines [26,27]. Additionally, the same PYT and AYTs can be used to predict advancements from the PYT and AYT across breeding cycles or within the same breeding cycle $[9,24]$. 


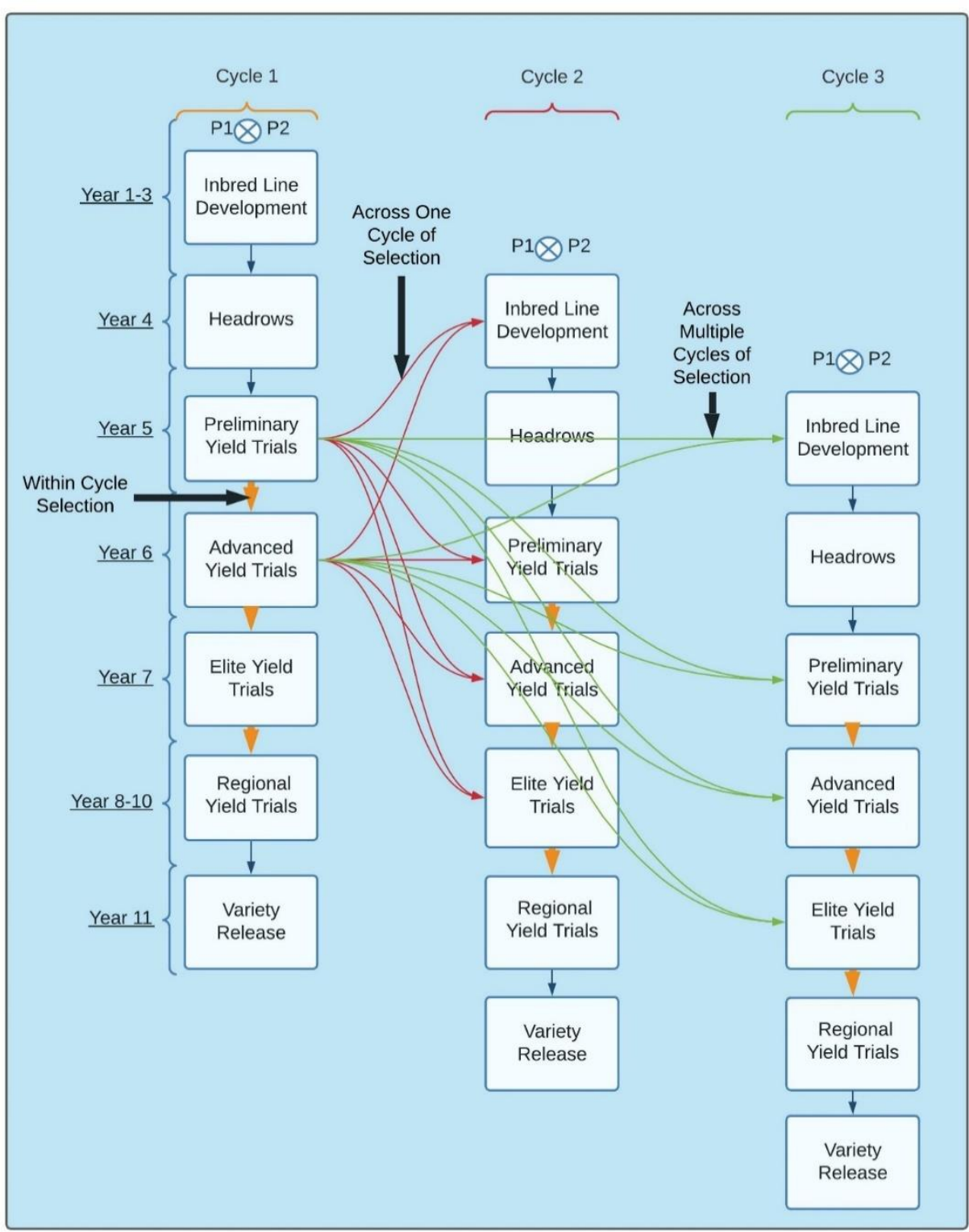

Figure 3. The implementation of GS for within and across breeding cycles based on an 11year breeding program from parental crossing to variety release. The within cycle selection is shown in orange vertical arrows within cycles for advancement through various yield trials, such as using GS to predict the performance of lines for advancements from the preliminary yield trials to the advanced yield trials compared to the blue arrows for the traditional phenotypic selection. Across cycle selection across a single year is shown using red arrows using preliminary and advanced yield trials to predict inbred lines and yield trials from cycle 1 to cycle 2 . Green arrows show the across cycle selection across multiple breeding cycles and are accomplished similarly to selection across a single year, but can utilize historical data using multiple cycles for prediction purposes.

The implementation of GS for selection in PYTs has been extensively studied $[15,21,24,25]$. The PYT has been intuitively chosen because they are the first time that lines are yield tested and generally constitute the largest filtering stage before subjecting lines to resource-demanding replicated multi-location yield trials. Additionally, since the PYT is the first time that lines are generally grown in plots, the seed is limited, resulting in either one or two replications. Therefore, since there is a trade-off between the number of lines being tested and the number of replications, Endelman et al. [21] demonstrated that 
higher prediction accuracy for GS can be achieved in the PYT by utilizing an unbalanced field design across multiple locations rather than testing all lines in a single location.

As mentioned previously, within cycle selection can be implemented in most stages in the breeding program. Verges and Van Sanford [25] demonstrated by utilizing within cycle selection, the number of lines in the PYT can be increased by phenotyping half of the lines and using the results to predict the performance of the other half. However, according to Michel et al. [24], predicting across cycles rather than within cycles decreased protein yield and content bias. Additionally, utilizing phenotypes from all breeding cycles increased prediction accuracy compared to within-cycle accuracy [28-30]. Further, Belamkar et al. [15] showed that prediction accuracy could be increased by merging across and within cycle selection by combining half of the lines in the within cycle PYT with previous PYT phenotypes. Furthermore, due to the limitations of resources and replications coupled with the moderate prediction accuracy for various traits, GS could be implemented to altogether remove the phenotyping of PYT and select lines for advancements directly into replicated yield trials or reduce the number of years lines are in the replicated yield trials.

Regardless of the approach, the implementation of GS can be viewed as a tool to reduce costly phenotyping and allocate resources elsewhere. GS can help aid or replace disease nurseries or select traits that are dependent on the environment for variation [30]. Irrespective of the stage of implementation, design of the breeding program, or trait, simulations can be used to compare the implementation of GS within breeding programs [13]. Throughout this review, many of the studies used simulations, including the development of the two-part breeding strategy proposed by Gaynor et al. [19]. There are a number of plant breeding simulation software such as "QU-GENE" [31], "AlphaSim" [32], and "DeltaGen" [33]. In Gaynor et al. [19], AlphaSim was used due to its ease, flexibility, and ability to simulate both plant and animal breeding programs. Recently, AlphaSim was developed into an R package [34]. Plant breeders can now simulate the majority of breeding decisions and GS optimizations before dedicating limited resources to redesigning their breeding programs.

\section{Population Improvement}

Population improvement has the greatest opportunity to increase genetic gain by leveraging the major advantage GS has over PS, which is decreasing the cycle time for variety development [8]. The goal of PI is to minimize the selection cycle for identifying parents in the crossing block. GS is adequately poised to break the need for phenotyping new parents by using GEBVs and simply genotyping untested lines without inbreeding or further selection. Therefore, recurrent selection can be accomplished rapidly within a breeding program. As mentioned above, the two-part strategy proposed by Gaynor et al. [19] relies heavily on the recurrent selection scheme for PD.

As discussed previously, Gaynor et al. [19] outlined two strategies for recurrent selection in a two-part breeding program. The two strategies were recurrent selection based on selecting $\mathrm{F}_{1}$ to cross with new parents added to the training population-based on inbreds from PYT or based on selection out of headrows. The two-part program was accomplished by randomly dividing the parents' as male and female parents equally. Then, male sterility techniques were applied to conduct open pollination of the females. Seeds were randomly selected from each half-sib family to continue the selection cycle, and the other seeds were used to produce DH lines, with a reduction in DH lines compared to the conventional program. The DH lines were subsequently screened in the PD pipeline, and GS was applied in the PYT for inclusion to the training population, but not the crossing block. The two-part scheme with headrow selection was conducted similarly but with selection out of the headrows instead of PYT. To mitigate increases in genotyping costs, the twopart scheme with headrow selection needs a reduction of lines per cross. The two-part 
plus headrow selection outperformed the regular two-part breeding scheme in terms of genetic gain [19].

\subsection{Selection scheme}

The benefit of the two-part strategy is in large part due to the decrease in cycle time and subsequent increase in genetic gain. By implementing a recurrent selection scheme, the breeding program can achieve the shortest possible cycle time. Most recurrent selection has been based on simulations for theoretical GS strategies in breeding programs (Figure 4).

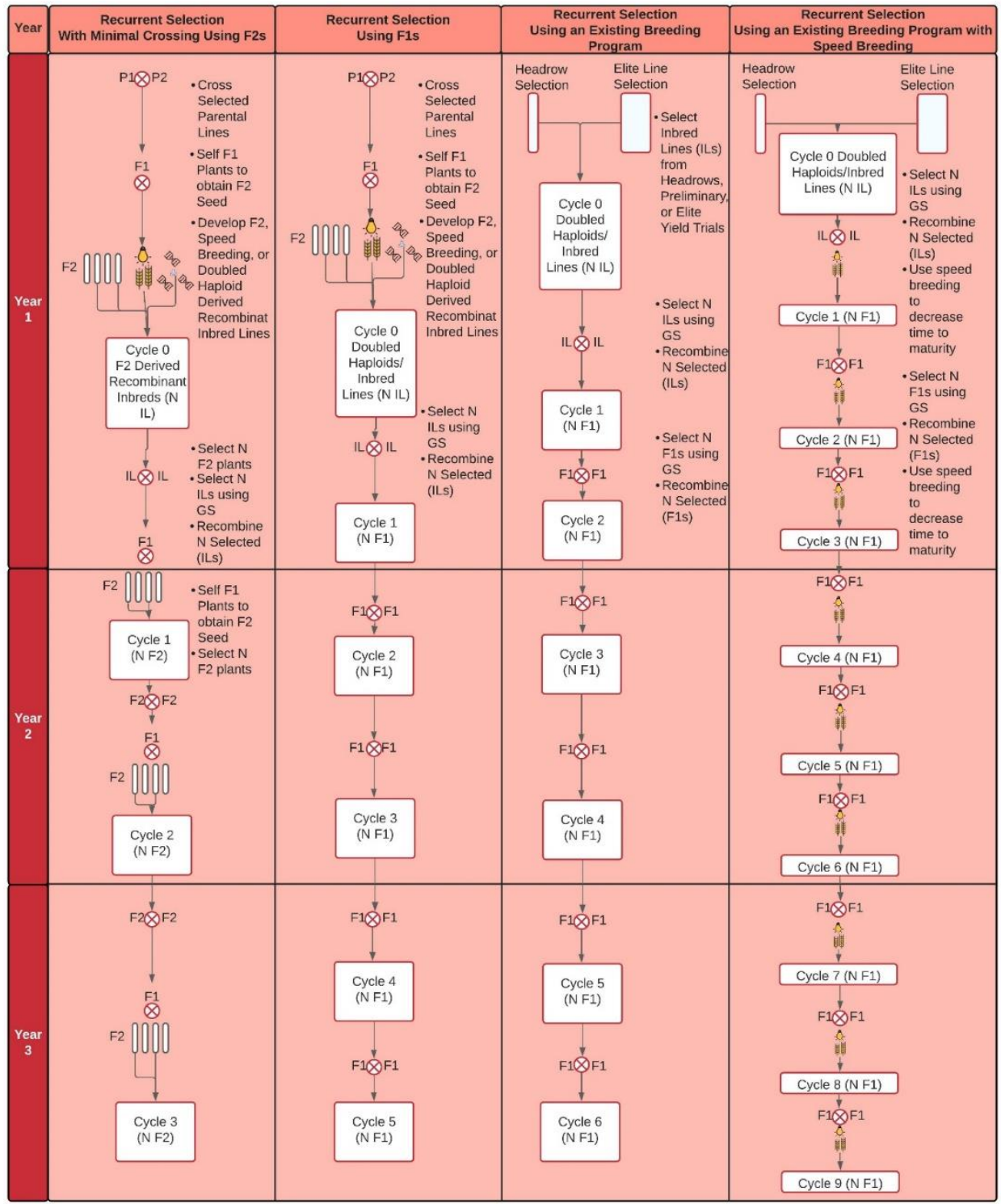

Figure 4. Genomic selection recurrent selection schemes comparing the number of recurrent selection cycles achieved over three years with $N$ being the number of selected recombinant inbred lines (IL), doubled-haploids (DH), F1, or $F_{2}$ lines. Recurrent selection with minimal crossing using $\mathrm{F}_{2}$ lines consists of developing an initial IL population and then implementing GS to select $N$ ILs to recombine, and the Fis are then self-pollinated to form an $\mathrm{F}_{2}$ population that makes up the next recurrent selection cycle. This method can achieve around one cycle per year. Recurrent selection using $F_{1 S}$ is similar to recurrent selection using $F_{2}$. However, the $F_{1 s}$ are not self-pollinated, and recurrent selection is implemented on the $\mathrm{F}_{1 \mathrm{~S}}$ to make up the next recurrent selection cycle and achieve up to two 
cycles per year. Further, the number of recurrent selection cycles can be increased by utilizing the existing breeding program and the use of speed breeding to decrease time to maturity for crossing and selection purposes.

In one of the first comparisons conducted for GS and marker-assisted recurrent selection (MARS) schemes, two cycles of selection for grain yield in maize (Zea mays L.) were simulated [35]. In the initial population development (Cycle 0), two inbred lines were crossed to form an $\mathrm{F}_{1}$ population that was developed into DHs. The DHs showed higher responses than $\mathrm{F}_{2}$ [35]. The best $\mathrm{DHs}$ were selected based on testcross performance. The selected DH lines were randomly mated to form $\mathrm{F}_{1 \mathrm{~s}}$ for Cycle 1. Genome-wide markers were used in GS, and significantly associated markers were used for MARS. Lines were selected using GS and MARS, and then randomly mated to form Cycle 2. This was repeated to form Cycle 3, where the response to selection was calculated. The simulations displayed that the maximum response was always greater with GS than MARS. Highly heritable traits displayed a 6\% increase with GS over MARS; however, for low heritable traits, the advantage increased to $18 \%$ for GS over MARS. Therefore, for a complex trait with low heritability, GS has a large advantage over MARS. Since selection indices have low heritability due to the combination of multiple traits, GS was further hypothesized to have an advantage over MARS when using selection indices.

Bernardo [36] outlined a GS scheme in inbred crops that minimizes crossing (Figure 4). Using the existing recombinant-inbred lines (RILs) from the breeding program that have been evaluated in multi-location year trials, the best RILs are selected based on their phenotypic performance. These selected RILs are crossed, and the $\mathrm{F}_{1}$ progenies were allowed to self-pollinate to $\mathrm{F}_{2}$. The $\mathrm{F}_{2}$ were then grown and genotyped with the best progeny selected based on GEBVs and crossed once again. These F1s are again self-pollinated to $\mathrm{F}_{2 \mathrm{~S}}$, and the process is repeated. Recurring selection ultimately decreases cycle time and increases genetic gain [36]. This type of crossing has also been called rapid cycling and focuses more on parental selection and crossing rather than later generational cultivar selection. In simulations, the recurrent selection scheme was compared to a recurrent selection scheme without self-pollination (Figure 4). Due to the time it takes to self-pollinate, three cycles of recurrent selection were accomplished in three years using a recurrent selection scheme with self-pollination compared to 5 cycles in three years using recurrent selection without self-pollination. The response to selection was 81 to $87 \%$ lower than a recurrent selection scheme without self-pollination. However, after three cycles, the response to selection with the recurrent selection with self-pollination improved 4 to $12 \%$ over the response to selection of the recurrent selection without self-pollination. The increase of response to selection for the self-pollination scheme was due to the increase of genetic variation from self-pollinating $\mathrm{F}_{1}$ to $\mathrm{F}_{2}$ that can then be exploited by selection. However, the simulations also determined the number of individuals evaluated in the self-pollination scheme should be twice as large as in the scheme without self-pollination. Further, increasing the selection intensity increased the response to selection. In self-pollinated crops with hand crossing, obtaining large amounts of seed is difficult, and therefore, the selection response is reduced by $\sim 20 \%$ compared to selection without self-pollination. However, this can be overcome with an increase in selection intensity and an increase in plants evaluated.

However, recurrent selection can be accomplished in the $\mathrm{F}_{1}$. For example, in intermediate wheatgrass (Thinopyrum intermedium), recurrent selection on $\mathrm{F}_{1}$ s has been proposed to aid in rapid domestication and improvement. The intermediate wheatgrass program started in 1988 with two cycles of PS in for seed size and fertility [37]. It was projected that it would take 20 years to reach the same level of genetic gain of wheat and 100 years to reach the same size [38]. Therefore, a GS scheme with a cycle time of one year was proposed. The proposed scheme starts with $\mathrm{F}_{1}$ seeds from the parent crosses. The $\mathrm{F}_{1}$ lines were then genotyped, and GS was used to determine the best $100 \mathrm{~F}_{1}$, which were then included in the crossing block for the next cycle. A total of 1,000 to 1,200 plants are then planted in the field for phenotypic evaluation to update the GS model. The recurrent selection 
scheme was estimated to increase the rate of genetic gain 2.6 times higher than PS for spike yield and higher for other traits.

The increase in genetic gain between the two-part breeding schemes and the conventional scheme is based on reducing cycle time. In the two-part breeding scheme, two cycles of recurrent selection were accomplished within a single year (Figure 4). The genetic gain can be further improved by increasing the number of cycles per year and further decreasing cycle time. Using rapid cycling via speed breeding, the length of cycle time could be drastically decreased with up to six cycles having been achieved [39]. However, a decrease in the accuracy of GS models and a reduction in genotypic variance can reduce genetic gains while increasing cycles of selection. Further, since GS requires genotypic data, every implementation of GS, especially in recurrent selection and PI, is dependent on the amount of time it takes to genotype the lines. To mitigate this, Hickey et al. [40] recommended that GS could be applied every two or three generations but would need to be supplemented with MAS or PS. Therefore, to maximize the number of recurrent selection cycles implemented with GS, the generation of genotyping, predicting, and crossing needs to be optimized [16].

\subsection{Integration of germplasm and maintaining genetic variance}

Another limitation of the two-part simulations in Gaynor et al. [19] is the division between the PI and PD in which selected DH lines were only included in the training population but not in the crossing block. In a practical implementation of the two-part strategy, the PI pipeline would become a pre-breeding scheme to rapidly produce lines for the PD pipeline. Further, the inbred lines from the PYT or headrows would be introduced into the crossing block as parents. The two-part system needs to integrate germplasm and parents from outside the program or via the PD pipeline. Further, within recurrent selection, methods for maintaining genetic variation need to be considered and implemented, as discussed in Rutkoski et al. [5]. This is even more important in a practical implementation of the two-part strategy where enough variation is needed to be maintained for selecting multiple traits throughout the PD pipeline. Genetic variation can be maintained by integrating outside germplasm or through optimal contribution and cross selection. Gaynor et al. [19] suggested that new integrations into the PD pipeline be used as males in order to spread their alleles throughout the population efficiently. Additionally, optimal contribution selection can be integrated to ensure newly introduced alleles are not lost [41,42]. As an approach, optimal contribution selection was developed to balance the contribution and mating of breeding parents for the next cycle to minimize genetic relatedness and inbreeding among parents and progeny [41]. In breeding programs such as those of CIMMYT, Mexico, the maintenance of genetic diversity is vital for the ability to screen and select lines in developing countries [43]. Optimal cross selection was simulated over a 20-year period and compared against traditional truncation selection with various numbers of cycles per year [44]. The optimal contribution selection utilized AlphaMate to account for optimized selection, genetic diversity, and cross-allocation altogether [44,45]. Using AlphaMate, optimal cross selection increased long-term genetic gain by up to $78 \%$ compared to truncation selection on a small number of parents. In addition, decrease of GS accuracy due to the loss of genetic relationship between the training and prediction populations was mitigated [44]. The ability to predict the performance of crosses increases the efficiency of genetic and financial resources, and therefore is a vital part of redesigning a breeding program to focus on GS and population development [43] 


\subsection{Optimal cross-prediction}

AlphaMate has the flexibility to be used in both cross and self-pollinated plant species. The software optimizes selection for genetic gain and genetic variation by minimizing group co-ancestry, inbreeding of individual mating, and maximizing group inbreeding. In addition to optimizing selection, breeding programs need to balance short and long-term genetic gain and individual contributions while maintaining genetic diversity [45]. Another software that accounts for the genetic variance for cross-prediction in biparental breeding populations is the "PopVar" package [46] in R. When predicting a cross, it was observed that the mean of the population explained more variation for the mean of the superior progeny than the genetic variance [46]. Likewise, the relevance of the mean was shown in Lado et al. [47] and Yao et al. [48], indicating that the mean of the population was more influential than the genetic variance when predicting crosses for grain yield in wheat. However, the importance of genetic variance was larger in end-use quality traits [47]. Additionally, genetic variance becomes more influential when predicting crosses when the difference in mean of the parents is low, as in the case when crossing elite material. Therefore, it is important to understand the parental materials when predicting and selecting crosses. PopVar predicts the genetic variance by measuring the variance among GEBVs, as outlined in Bernardo [49]. In addition, utilizing PopVar or other crossing simulations in recurrent selection and population development allows the imposition of lower selection intensity while maintaining genetic variation to increase genetic gain rapidly.

To maximize the effect of a recurrent selection scheme, methods to select multiple traits efficiently must be developed, including, in part, selection index or separate focus on single trait improvement. For example, Yao et al. [48] showed the use of selection indices using a usefulness rating for grain yield and end-use quality traits composed of weighted values for grain yield, extensibility, and maximum resistance, and demonstrated the ability to improve grain yield and end-use quality in wheat simultaneously. Additionally, near-zero or positive correlations were identified in predicted progeny for negatively correlated traits in the parents [48]. PopVar can predict values for correlated traits, which allows simultaneous selection of traits. In addition, PopVar predicted correlated responses of multiple traits, such as with the negative correlation between grain yield and deoxynivalenol [46]. In general, when selecting multiple traits, there is a tradeoff on the performance of either trait or of both. Nevertheless, PopVar can identify crosses that have a favorable mean for one, with a near-zero mean in the other, or can determine a favorable mean for both, as observed previously [48]. Therefore, PopVar can allow for genetic gain for simultaneous selection, even among negatively correlated traits.

In both Yao et al. [48] and Mohammadi et al. [46], common GS models were used to predict marker effects for parents. The cross predictions utilizing GEBVs were laid out in Endelman [50] using rrBLUP. Additionally, the R package "sommer" [51] can use univariate and multivariate linear mixed models. The "sommer" package has the added capability of integrating dominance and epistatic effects, which can be utilized for hybrid selection. For population development, additive effects are more important. Therefore, there is a greater emphasis on utilizing additive effects for sustained genetic gain [46].

Recurrent selection is dependent on identifying parents through the developed inbred lines or the progeny of crosses such as $\mathrm{F}_{1}$, or after self-pollination using the $\mathrm{F}_{2}$ lines, depending on the recurrent selection scheme. Utilizing GS in parental selection allows the reduction in cycle time, and therefore increases genetic gain. Inbred lines can be selected based on traditional self-pollination or DH production described previously. However, inbred lines for parental selection are not needed for the implementation of GS and are an artifact of conventional breeding programs. The selection of $F_{1}$ or $F_{2}$ in a recurrent selection scheme have been reported in both empirical and simulation studies $[5,35,36]$. By selecting non-inbred lines, the cycle time can be drastically reduced, and recurrent selection by means of genotyping, genomic selection, and then crossing can be repeated for rapid cycling, and thus accomplish rapid recurrent selection. A common adage in plant breeding 
is "Plant breeding is a numbers game". This adage relates to the breeder's equation for genetic gain in which increasing the number of lines increases genetic variance and selection intensity. However, breeding programs have limited resources and can only screen so many lines. Therefore, the selection of parents for crossing needs to be efficient by making fewer crosses among the parental lines and effectively changes plant breeding as a "numbers game" to a more precise "chess game." For parental selection, the ability to identify the breeding populations with the highest mean performance prior to making a cross is more important than the number and size of the breeding population [52,53]. Additionally, with better parental selection, the family size of the population can be increased with a decrease in the number of overall populations. Further, Hickey et al. [54] showed an increase in prediction accuracy with the increase of lines within families, whereas Verges and Van Sanford [25] concluded a minimum number of 25 lines per family were needed to stabilize prediction accuracies at the PYT level.

\section{Real world applications}

The first recurrent GS experiment in crops was conducted for grain yield and stover quality in maize [4], where GS was compared with MARS (Table 3). In contrast to GS, MARS is based on MAS with significant markers for recurrent selection. A total of three cycles of selection for grain yield and stover quality in maize was compared in the recurrent selection scheme. The scheme started with an initial biparental population (Cycle 0) which was evaluated for traits and genotyped for markers of interest [4]. Multi-trait selection indices were then constructed for grain yield and stover quality. Cycle 0 individuals were ranked according to selection index values, with the top individuals selected and intermated to form Cycle 1. Cycle 1 individuals were genotyped and used to predict the selection index. The Cycle 1 individuals were once again ranked for their predicted values, with the top individuals selected and intermated to form Cycle 2. This was completed one more time to produce Cycle 3. Individuals from Cycles 0, 1, 2, and 3 were compared and evaluated in field trials. GS displayed a higher stover index and grain yield in Cycle 3 compared to MARS. Additionally, the yield index for Cycles 2 and 3 was significantly higher than Cycle 1 for GS but not for MARS. Further, the majority of gains were accomplished from Cycle 1 due to Cycle 0 selection being based on PS. Overall, GS resulted in a greater genetic gain than MARS for selection indices. However, for single traits, the majority of Cycle 2 selections for GS and MARS showed no improvement over Cycle 1. Further, grain yield showed no improvement over Cycle 0. 
Table 3. Empirical genomic selection (GS) recurrent selection studies.

\begin{tabular}{|c|c|c|c|c|c|}
\hline Crop & Trait & Cycles & $\begin{array}{l}\text { Selection } \\
\text { methods } \\
\text { compared }{ }^{1}\end{array}$ & Gain & Reference \\
\hline Maize & $\begin{array}{l}\text { Stover Index } \\
\text { and Grain } \\
\text { Yield }\end{array}$ & 3 & MARS vs GS & $\begin{array}{l}\text { GS had higher genetic } \\
\text { gain compared to MARS }\end{array}$ & {$[4]$} \\
\hline Wheat & $\begin{array}{l}\text { Quantitative } \\
\text { Adult Plant } \\
\text { Stem Rust } \\
\text { Resistance }\end{array}$ & 2 & PS vs GS & $\begin{array}{c}\text { GS had equal rates of } \\
\text { genetic gain compared to } \\
\text { PS }\end{array}$ & [5] \\
\hline Wheat & Grain Yield & 1 & GS models & $\begin{array}{c}\text { Reproducing kernel } \\
\text { Hilber Spaces (RKHS) GS } \\
\text { model had the highest } \\
\text { realized genetic gain }\end{array}$ & [43] \\
\hline Barley & $\begin{array}{l}\text { Grain Yield } \\
\text { and DON }\end{array}$ & 3 & $\begin{array}{c}\mathrm{TP} \\
\text { optimization }\end{array}$ & $\begin{array}{l}\text { Optimization algorithms } \\
\text { improved accuracy } \\
\text { compared to randomly } \\
\text { selected TPs }\end{array}$ & [27] \\
\hline Wheat & $\begin{array}{c}\text { Wheat Grain } \\
\text { Fructan }\end{array}$ & 2 & $\begin{array}{l}\text { GS with TS vs } \\
\text { GS with (OCS) }\end{array}$ & $\begin{array}{l}\text { OCS and TS had similar } \\
\text { genetic gains; OCS } \\
\text { retained greater genetic } \\
\text { variance }\end{array}$ & [55] \\
\hline
\end{tabular}

${ }^{1}$ GS- Genomic selection; MARS- Marker-assisted recurrent selection; OCS- Optimal contribution selection; PS- Phenotypic selection; TP- Training population; TS- Truncation selection

Rutkoski et al. [5] reported the first realized GS in wheat and the first using recurrent selection (Table 3), where two cycles of GS to one cycle of PS for quantitative adult plant resistance to stem rust were compared. Accordingly, GS leads to equal rates of genetic gain per unit time compared to PS, but rapidly decreased genetic variance. The low prediction accuracy in the first cycle of GS led to the lack of increase in genetic gain over PS. CIMMYT has also conducted empirical GS in early generations [43], where $200 \mathrm{~F}_{2}$ plants were selected from 40 different crosses (Table 3). The $\mathrm{F}_{2: 4}$ lines were bulked and tested in a replicated yield trial for two years. Results showed that the RKHS model had the highest accuracy with an increase in grain yield of $7 \%$ when comparing the highest GEBV lines to the lowest GEBV lines [43]. Additionally, recurrent selection has been applied in other public breeding programs. For instance, three cycles of selection in a spring six-row barley population were able to increase genetic gains for both grain yield and deoxynivalenol, which are two negatively correlated traits [27]. Further, recurrent selection implemented with optimized contribution selection increased wheat grain fructan content by $34 \%$ while controlling the rate of inbreeding [55]. Altogether, previous simulations and actual field validation studies showed recurrent selection using GS to increase genetic gain. Therefore, there can be a significant improvement in gains achieved through selection in both of private and public breeding programs through implementation of GS and a two-part breeding program. 


\section{Conclusions}

In our review, we explored redesigning a breeding program for genomic selection and the implementation of the two-part breeding scheme. The two-part breeding program was proposed to differentiate between PI and PD. The PD pipeline represents the traditional breeding program based on PS for variety release. GS models now allow breeders to select lines for implementing into the crossing block from virtually any stage of the breeding program and therefore drastically reduce the cycle time for parental selection. Additionally, GS can be implemented to predict lines for advancement within and across breeding cycles and years. Further, by implementing recurrent selection into PI, the genetic gain is effectively limited by the speed and ability to genotype, predict, and cross parental lines and $F_{1}$. Therefore, by utilizing GS and reducing the reliance on PS alone, breeders can redesign their breeding programs and increase genetic gains. This increase in genetic gains will help improve lines and meet the demands of a growing population. Now it is up to breeders to implement these changes and prove the power of GS approaches.

Author Contributions: Conceptualization, L.F.M.; writing-original draft preparation, L.F.M., A.W.H., and K.S.S.; writing - review and editing, L.F.M., A.W.H., K.S.S., D.N.L., and A.H.C.; supervision, A.H.C.; funding acquisition, A.H.C.

Funding: This research was partially funded by the National Institute of Food and Agriculture (NIFA) of the U.S. Department of Agriculture (Award number 2016-68004-24770 and 2022-6801336439), Hatch project 1014919, and the O.A. Vogel Research Foundation at Washington State University.

Data Availability Statement: Not applicable

Conflicts of Interest: The authors declare no conflict of interest. 


\section{References}

1. Acquaah, G. Principles of Plant Genetics and Breeding; Second edition.; Hoboken, New Jersey : Wiley-Blackwell: Hoboken, New Jersey, 2012;

2. Varshney, R.K.; Roorkiwal, M.; Sorrells, M.E. Genomic Selection for Crop Improvement: New Molecular Breeding Strategies for Crop Improvement; Springer, 2017; ISBN 3-319-63170-5.

3. Carver, B.F. Wheat: Science and Trade; Ames, Iowa : Wiley-Blackwell: Ames, Iowa, 2009;

4. Massman, J.M.; Jung, H.-J.G.; Bernardo, R. Genomewide Selection versus Marker-Assisted Recurrent Selection to Improve Grain Yield and Stover-Quality Traits for Cellulosic Ethanol in Maize. Crop Sci. 2013, 53, 58, doi:10.2135/cropsci2012.02.0112.

5. Rutkoski, J.; Singh, R.P.; Huerta-Espino, J.; Bhavani, S.; Poland, J.; Jannink, J.L.; Sorrells, M.E. Genetic Gain from Phenotypic and Genomic Selection for Quantitative Resistance to Stem Rust of Wheat. Plant Genome 2015, 8, 0, doi:10.3835/plantgenome2014.10.0074.

6. Meuwissen, T.H.E.; Hayes, B.J.; Goddard, M.E. Prediction of Total Genetic Value Using Genome-Wide Dense Marker Maps. Genetics 2001, 157, 1819-1829, doi:https://doi.org/10.1534/genetics.116.189795.

7. Heffner, E.L.; Sorrells, M.E.; Jannink, J.-L. Genomic Selection for Crop Improvement. Crop Sci. 2009, 49, 1, doi:10.2135/cropsci2008.08.0512.

8. Heffner, E.L.; Lorenz, A.J.; Jannink, J.-L.; Sorrells, M.E. Plant Breeding with Genomic Selection: Gain per Unit Time and Cost. Crop Sci. 2010, 50, 1681, doi:10.2135/cropsci2009.11.0662.

9. Michel, S.; Ametz, C.; Gungor, H.; Akgöl, B.; Epure, D.; Grausgruber, H.; Löschenberger, F.; Buerstmayr, H. Genomic Assisted Selection for Enhancing Line Breeding: Merging Genomic and Phenotypic Selection in Winter Wheat Breeding Programs with Preliminary Yield Trials. Theor. Appl. Genet. 2017, 130, 363-376, doi:10.1007/s00122016-2818-8.

10. Falconer, D.S.; Mackay, T.F.C. Introduction to Quantitative Genetics. 1996. Harlow Essex UK Longmans Green 1996, 3 .

11. Cobb, J.N.; Juma, R.U.; Biswas, P.S.; Arbelaez, J.D.; Rutkoski, J.; Atlin, G.; Hagen, T.; Quinn, M.; Ng, E.H. Enhancing the Rate of Genetic Gain in Public-Sector Plant Breeding Programs: Lessons from the Breeder's Equation. Theor. Appl. Genet. 2019, 132, 627-645, doi:10.1007/s00122-019-03317-0.

12. Lorenzana, R.E.; Bernardo, R. Accuracy of Genotypic Value Predictions for Marker-Based Selection in Biparental Plant Populations. Theor. Appl. Genet. 2009, 120, 151-161, doi:10.1007/s00122-009-1166-3.

13. Bernardo, R. Breeding for Quantitative Traits in Plants; 3rd ed.; Stemma Press: Woodbury, Minnesota, 2020; ISBN 978-0-9720724-3-4.

14. Arruda, M.P.; Lipka, A.E.; Brown, P.J.; Krill, A.M.; Thurber, C.; Brown-Guedira, G.; Dong, Y.; Foresman, B.J.; Kolb, F.L. Comparing Genomic Selection and Marker-Assisted Selection for Fusarium Head Blight Resistance in Wheat (Triticum Aestivum L.). Mol. Breed. 2016, 36, 84, doi:10.1007/s11032-016-0508-5.

15. Belamkar, V.; Guttieri, M.J.; Hussain, W.; Jarquín, D.; El-basyoni, I.; Poland, J.; Lorenz, A.J.; Baenziger, P.S. Genomic Selection in Preliminary Yield Trials in a Winter Wheat Breeding Program. G3amp58 GenesGenomesGenetics 2018, 8, 2735-2747, doi:10.1534/g3.118.200415.

16. Bassi, F.M.; Bentley, A.R.; Charmet, G.; Ortiz, R.; Crossa, J. Breeding Schemes for the Implementation of Genomic Selection in Wheat ( Triticum Spp . ). Plant Sci. 2016, 242, 23-36, doi:10.1016/j.plantsci.2015.08.021.

17. Robertsen, C.D.; Hjortshøj, R.L.; Janss, L.L. Genomic Selection in Cereal Breeding. Agronomy 2019, 9, 95, doi:10.3390/agronomy9020095.

18. Larkin; Lozada; Mason Genomic Selection-Considerations for Successful Implementation in Wheat Breeding Programs. Agronomy 2019, 9, 479, doi:10.3390/agronomy9090479. 
19. Gaynor, R.C.; Gorjanc, G.; Bentley, A.R.; Ober, E.S.; Howell, P.; Jackson, R.; Mackay, I.J.; Hickey, J.M. A Two-Part Strategy for Using Genomic Selection to Develop Inbred Lines. Crop Sci. 2017, 57, 2372-2386, doi:10.2135/cropsci2016.09.0742.

20. Rutkoski, J.E.; Poland, J.A.; Singh, R.P.; Huerta-Espino, J.; Bhavani, S.; Barbier, H.; Rouse, M.N.; Jannink, J.-L.; Sorrells, M.E. Genomic Selection for Quantitative Adult Plant Stem Rust Resistance in Wheat. Plant Genome 2014, 7, 0, doi:10.3835/plantgenome2014.02.0006.

21. Endelman, J.B.; Atlin, G.N.; Beyene, Y.; Semagn, K.; Zhang, X.; Sorrells, M.E.; Jannink, J.-L. Optimal Design of Preliminary Yield Trials with Genome-Wide Markers. Crop Sci. 2014, 54, 48-59, doi:10.2135/cropsci2013.03.0154.

22. Hoefler, R.; González-Barrios, P.; Bhatta, M.; Nunes, J.A.R.; Berro, I.; Nalin, R.S.; Borges, A.; Covarrubias, E.; DiazGarcia, L.; Quincke, M.; et al. Do Spatial Designs Outperform Classic Experimental Designs? J. Agric. Biol. Environ. Stat. 2020, 25, 523-552, doi:10.1007/s13253-020-00406-2.

23. Longin, C.F.H.; Mi, X.; Würschum, T. Genomic Selection in Wheat: Optimum Allocation of Test Resources and Comparison of Breeding Strategies for Line and Hybrid Breeding. Theor. Appl. Genet. 2015, 128, 1297-1306, doi:10.1007/s00122-015-2505-1.

24. Michel, S.; Ametz, C.; Gungor, H.; Epure, D.; Grausgruber, H.; Löschenberger, F.; Buerstmayr, H. Genomic Selection across Multiple Breeding Cycles in Applied Bread Wheat Breeding. Theor. Appl. Genet. 2016, 129, 11791189, doi:10.1007/s00122-016-2694-2.

25. Verges, V.L.; Van Sanford, D.A. Genomic Selection at Preliminary Yield Trial Stage: Training Population Design to Predict Untested Lines. Agronomy 2020, 10, 60, doi:10.3390/agronomy10010060.

26. Michel, S.; Kummer, C.; Gallee, M.; Hellinger, J.; Ametz, C.; Akgöl, B.; Epure, D.; Löschenberger, F.; Buerstmayr, H. Improving the Baking Quality of Bread Wheat by Genomic Selection in Early Generations. Theor. Appl. Genet. 2018, 131, 477-493, doi:10.1007/s00122-017-2998-x.

27. Tiede, T.; Smith, K.P. Evaluation and Retrospective Optimization of Genomic Selection for Yield and Disease Resistance in Spring Barley. Mol. Breed. 2018, 38, 1-16.

28. Auinger, H.-J.; Schönleben, M.; Lehermeier, C.; Schmidt, M.; Korzun, V.; Geiger, H.H.; Piepho, H.-P.; Gordillo, A.; Wilde, P.; Bauer, E.; et al. Model Training across Multiple Breeding Cycles Significantly Improves Genomic Prediction Accuracy in Rye (Secale Cereale L.). Theor. Appl. Genet. 2016, 129, 2043-2053, doi:10.1007/s00122-0162756-5.

29. Merrick, L.F.; Carter, A.H. Comparison of Genomic Selection Models for Exploring Predictive Ability of Complex Traits in Breeding Programs. Plant Genome 2021, n/a, e20158, doi:10.1002/tpg2.20158.

30. Merrick, L.F.; Burke, A.B.; Chen, X.; Carter, A.H. Breeding With Major and Minor Genes: Genomic Selection for Quantitative Disease Resistance. Front. Plant Sci. 2021, 12, 1599, doi:10.3389/fpls.2021.713667.

31. Podlich, D.W.; Cooper, M. QU-GENE: A Simulation Platform for Quantitative Analysis of Genetic Models. Bioinforma. Oxf. Engl. 1998, 14, 632-653.

32. Faux, A.-M.; Gorjanc, G.; Gaynor, C.; Battagin, M.; Edwards, S.M.; Wilson, D.L.; Hearne, S.; Gonen, S.; Hickey, J.M. AlphaSim: Software for Breeding Program Simulation. 2016.

33. Jahufer, M.Z.Z.; Luo, D. DeltaGen: A Comprehensive Decision Support Tool for Plant Breeders. Crop Sci. 2018, 58, 1118-1131.

34. Gaynor, R.C.; Gorjanc, G.; Hickey, J.M. AlphaSimR: An R Package for Breeding Program Simulations. G3 2021, 11, jkaa017.

35. Bernardo, R.; Yu, J. Prospects for Genomewide Selection for Quantitative Traits in Maize. Crop Sci. 2007, 47, 1082, doi:10.2135/cropsci2006.11.0690. 
36. Bernardo, R. Genomewide Selection with Minimal Crossing in Self-Pollinated Crops. Crop Sci. 2010, 50, 624, doi:10.2135/cropsci2009.05.0250.

37. Crain, J.; Bajgain, P.; Anderson, J.; Zhang, X.; DeHaan, L.; Poland, J. Enhancing Crop Domestication through Genomic Selection, a Case Study of Intermediate Wheatgrass. Front. Plant Sci. 2020, 11, 319.

38. DeHaan, L.R.; Wang, S.; Larson, S.; Cattani, D.J.; Zhang, X.I.; Kantarski, T.; Batello, C.; Wade, L.; Cox, S.; Pogna, N. Current Efforts to Develop Perennial Wheat and Domesticate Thinopyrum Intermedium as a Perennial Grain. In Proceedings of the Perennial crops for food security: Proc. of the FAO Expert Workshop; 2014.

39. Watson, A.; Ghosh, S.; Williams, M.J.; Cuddy, W.S.; Simmonds, J.; Rey, M.-D.; Asyraf Md Hatta, M.; Hinchliffe, A.; Steed, A.; Reynolds, D.; et al. Speed Breeding Is a Powerful Tool to Accelerate Crop Research and Breeding. Nat. Plants 2018, 4, 23-29, doi:10.1038/s41477-017-0083-8.

40. Hickey, L.T.; N. Hafeez, A.; Robinson, H.; Jackson, S.A.; Leal-Bertioli, S.C.M.; Tester, M.; Gao, C.; Godwin, I.D.; Hayes, B.J.; Wulff, B.B.H. Breeding Crops to Feed 10 Billion. Nat. Biotechnol. 2019, 37, 744-754, doi:10.1038/s41587019-0152-9.

41. Meuwissen, T.H.E. Maximizing the Response of Selection with a Predefined Rate of Inbreeding. J. Anim. Sci. 1997, 75, 934-940.

42. Woolliams, J. a.; Berg, P.; Dagnachew, B. s.; Meuwissen, T. h. e. Genetic Contributions and Their Optimization. J. Anim. Breed. Genet. 2015, 132, 89-99, doi:10.1111/jbg.12148.

43. Dreisigacker, S.; Crossa, J.; Pérez-Rodríguez, P.; Montesinos-Loópez, O.A.; Rosyara, U.; Juliana, P.; Mondal, S.; Crespo-Herrera, L.; Govindan, V.; Singh, R.P. Implementation of Genomic Selection in the CIMMYT Global Wheat Program, Findings from the Past 10 Years. Crop Breed. Genet. Genomics 2021, 3.

44. Gorjanc, G.; Gaynor, R.C.; Hickey, J.M. Optimal Cross Selection for Long-Term Genetic Gain in Two-Part Programs with Rapid Recurrent Genomic Selection. Theor. Appl. Genet. 2018, 131, 1953-1966.

45. Gorjanc, G.; Hickey, J.M. AlphaMate: A Program for Optimizing Selection, Maintenance of Diversity and Mate Allocation in Breeding Programs. Bioinformatics 2018, 34, 3408-3411.

46. Mohammadi, M.; Tiede, T.; Smith, K.P. PopVar: A Genome-Wide Procedure for Predicting Genetic Variance and Correlated Response in Biparental Breeding Populations. Crop Sci. 2015, 55, 2068, doi:10.2135/cropsci2015.01.0030.

47. Lado, B.; Battenfield, S.D.; Guzmán, C.; Quincke, M.; Singh, R.P.; Dreisigacker, S.; Peña-Bautista, R.J.; Fritz, A.K.; Silva, P.; Poland, J. Strategies for Selecting Crosses Using Genomic Prediction in Two Wheat Breeding Programs. 2017.

48. Yao, J.; Zhao, D.; Chen, X.; Zhang, Y.; Wang, J. Use of Genomic Selection and Breeding Simulation in Cross Prediction for Improvement of Yield and Quality in Wheat (Triticum Aestivum L.). Crop J. 2018, 6, 353-365, doi:10.1016/j.cj.2018.05.003.

49. Bernardo, R. Genomewide Selection of Parental Inbreds: Classes of Loci and Virtual Biparental Populations. Crop Sci. 2014, 54, 2586-2595, doi:10.2135/cropsci2014.01.0088.

50. Endelman, J.B. Ridge Regression and Other Kernels for Genomic Selection with R Package RrBLUP. Plant Genome J. 2011, 4, 250, doi:10.3835/plantgenome2011.08.0024.

51. Covarrubias-Pazaran, G. Software Update: Moving the R Package Sommer to Multivariate Mixed Models for GenomeAssisted Prediction; 2018; p. 354639;.

52. Bernardo, R. Parental Selection, Number of Breeding Populations, and Size of Each Population in Inbred Development. Theor. Appl. Genet. 2003, 107, 1252-1256, doi:10.1007/s00122-003-1375-0.

53. Witcombe, J.R.; Gyawali, S.; Subedi, M.; Virk, D.S.; Joshi, K.D. Plant Breeding Can Be Made More Efficient by Having Fewer, Better Crosses. BMC Plant Biol. 2013, 13, 22, doi:10.1186/1471-2229-13-22. 
54. Hickey, J.M.; Dreisigacker, S.; Crossa, J.; Hearne, S.; Babu, R.; Prasanna, B.M.; Grondona, M.; Zambelli, A.; Windhausen, V.S.; Mathews, K.; et al. Evaluation of Genomic Selection Training Population Designs and Genotyping Strategies in Plant Breeding Programs Using Simulation. Crop Sci. 2014, 54, 1476-1488, doi:10.2135/cropsci2013.03.0195.

55. Veenstra, L.D.; Poland, J.; Jannink, J.-L.; Sorrells, M.E. Recurrent Genomic Selection for Wheat Grain Fructans. Crop Sci. 2020, 60, 1499-1512. 